\title{
Improving on Symbolic Learning System Based on Genetic Algorithm
}

\author{
Limei Feng Xizhao Wang \\ Machine Learning Center, Faculty of Mathematics and Computer Science, Hebei University, Baoding 071002, P. R. \\ China
}

\begin{abstract}
This paper uses GAssist system to get symbolic rules and proposes four techniques to improve it. A new population initialization method is applied and fitness scaling is used to promote the population's convergence. It also improves the deserted hierarchical selection operator and combines it with the MDLbased fitness function to control bloat effect. Finally, a new stop criterion to GA is studied. The experimental results show that the system is further improved. Comparing with other systems GA system is roughly comparable in generalization capacity but the efficiency needs improve.
\end{abstract}

Keywords: GAssist system, Population initialization method, Fitness scaling, Hierarchical selection operator, New stop criterion

\section{Introduction}

Symbolic learning is to induce rule sets from symbolic datasets. The values of symbolic datasets are discrete, unordered and don't have operation relation among values. Continuous datasets can be converted to symbolic ones by discretizing algorithm. Therefore, methods of symbolic learning are very practical and could be widely used. Research on symbolic learning has very important meaning. Using GA to search rule sets is one of the most important methods for symbolic learning problems.

An example of the use of GA for symbolic learning is GABIL system [1]-[2] described by DeJong, K. A.and Spears, W. M. A chromosome represents a rule sets and the length of it is variable. GAssist system is an improved edition of GABIL system [3]. A new smart crossover operator is proposed by Jaume Bacardit [4]. The operator selects more than two parents and merges the rules of them to make the final individual have the highest training accuracy. The ensemble technique Bagging is studied to combine with GAssist system. The tests show that the combination is very useful [5].

This paper aims at symbolic learning problems, thus continuous attributes are discretized by Kohonen algorithm [6]-[7] first. In order to make it convenient to compare, the system is called GAssist-Kohonen system.

Rule representation is introduced in section 2 . Section 3 describes a new population initialization method and section 4 describes the introduced fitness scaling. Section 5 introduces the original hierarchical selection operator first, and then describe the improvement on it in detail. Experimental results are shown in section 7 . Finally, a conclusion and future work are given.

\section{Rule representation}

The use of GA to solve symbolic learning problems is to seek a IF-THEN rule set that performs best on training examples. The learning process is to search a large space of candidate rule sets to get the one having the highest fitness. The hypothesis of GA is often encoded by bit strings. The candidate hypothesis space is determined by the encoding method. The encoding method of GAssist system is as follows:

- For a single attribute, a bit string is used to describe the value of it. For example, attribute A can take on three values $a_{1} 、 a_{2} 、 a_{3}$. Three bits are needed to represent it. Setting 1 represents the attribute can take the corresponding value. Then the string 010 indicates that the value of attribute $A$ is the second one $\mathrm{a}_{2}$.

- Given the bit string that represents a single attribulte, conjunctions of bit strings indicate the constrains on multiple attributes.

- The values of multiple attributes can be represented by conjunctions of the corresponding bit strings.

A rule is represented by the conjunction of the rule precondition bit string and the rule postcondition bit string.

Because the hypothesis often consists of more than one rule and a chromosome represent a rule set , the length of the chromosome is variable. 


\section{New population initialization method}

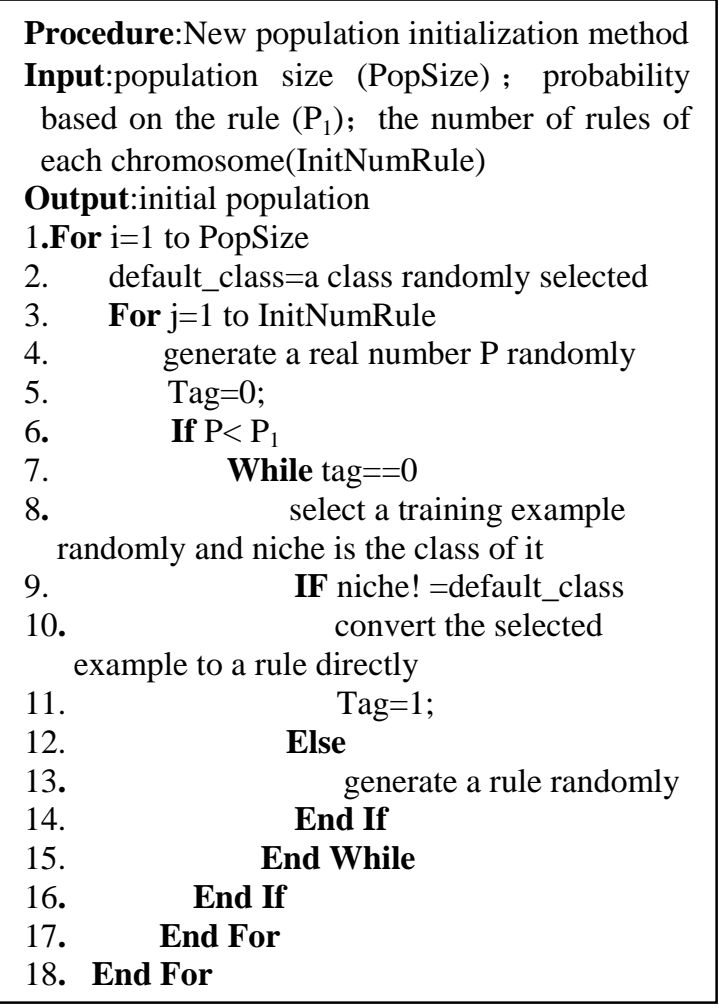

Fig. 1: New population initialization method.

The initial population is generated total randomly in GAssist system. At a later time, Jaume Bacardit proposed CWSI [8] (smart initialization operator with class-wise sampling) method. Experiments on 25 datasets indicates that the performance of CWSI is better on only one dataset. Therefore, the initial population is randomly generated in GAssist-Kohonen system that is the basic system for us to solve symbolic problems.

If the initial population is generated total randomly not considering the information of training examples the performance of the initial rule sets couldn't be very good . But if excessive information of training examples is used the generated rules may be too specific and the generalization capacity is also bad. Therefore, in this paper the rules of the initial population consist of two parts. One part is randomly generated and the other part is converted directly by exmples. The technique is different from CWSI. We use the probability $\mathrm{P}_{1}$ to control the proportion of the introduced training examples. For each rule of each chromosome, a real number is randomly generated. If it is smaller than $\mathrm{P}_{1}$, select an training example randomly and then convert it to a rule directly. If it is not small than $\mathrm{P}_{1}$, generate a rule randomly. GAssist system uses a static default rule mechanism [9], thus the class of the selected training example can't be the default class of the chromosome. The algorithm of the new initialization method is described in Figure 1.

\section{Introduction of fitness scaling}

In the earlier stage of evolution, if a few chromosomes has very high fitness values, they will be selected with higher probabilities. Thus, the diversity of the population is lost, which leads to premature convergence. In the later stage of evolution, the difference of chromosomes becomes smaller and the selection process is similar to randomly select. So, the evolution process becomes slow. In order to avoid the above two drawbacks, fitness scaling is introduced. Assume $\mathrm{f}$ is the original fitness value and $f$ ' is the new one [10], the formula of fitness scaling is as follows:

$$
f^{\prime}=a f+b
$$

where coefficients $\mathrm{a}$ and $\mathrm{b}$ is determined by the following two conditions :

$$
\begin{aligned}
& f_{\text {avg }}^{\prime}=f_{\text {avg }} \\
& f^{\prime} \text { max } \\
& =c \cdot f_{\text {avg }}
\end{aligned}
$$

where $c$ is the number of expected ones desired for the best chromosome. The new fitness value may be negative. In this case, the following formula is used.

$$
f_{\text {min }}^{\prime}=0
$$

The following inequation is first calculated when calculating the values of coefficients a and b:,

$$
f_{\min }>\frac{c \cdot f_{\text {avg }}-f_{\text {max }}}{c-1}
$$

if the above inequation is satisfied, then :

$$
\begin{aligned}
& a=\frac{f_{\text {avg }}}{f_{\text {avg }}-f_{\text {min }}} \\
& b=\frac{-f_{\text {avg }} \cdot f_{\text {min }}}{f_{\text {avg }}-f_{\text {min }}} f_{\text {avg }}
\end{aligned}
$$

if the above inequation is not satisfied, then :

$$
\begin{aligned}
& a=\frac{c-1}{f_{\text {max }}-f_{\text {avg }}} f_{\text {avg }} \\
& b=\frac{f_{\text {max }}-c \cdot f_{\text {avg }}}{f_{\text {max }}-f_{\text {avg }}} f_{\text {avg }}
\end{aligned}
$$




\section{Improving on hierarchical selection operator}

\subsection{Introduction of hierarchical selection operator}

The fitness function of GABIL system only considers the training accuracy of the chromosome and ignore the complexity factor, which leads to the system to improve the training accuracy by memorizing training examples. As a result, the length of the chromosome increases without control and the generalization capacity of the rule set is bad, which is called bloat effect [11].

GAssist system experienced a developing process. In order to control bloat effect, it ever used hierarchical selection operator. If the training accuracy difference of two chromosomes is small than the threshold setted in advance, the shorter one is selected first. If it is not small than the setted theshold, the chromosome having higher fitness is selected. At a later time, the MDL-based fitness function [12] is proposed, which has better performace than the hierarchical selection operator. Therefore, the hierarchical selection operator is deserted. However, it has the following two reasonable points:

- Consistant with the Occam's razor principle The hierarchical selection operator is integrated with the tournament selection operator. When the performance of two chromosomes is similar, the shorter one is selected first, which is consistant with the Occam's razor principle 。

- $\quad$ Specific-to-general policy

In the earlier stage of evolution, the training accuracy difference of chromosomes is large, the chromosome with higher training accuracy is selected, the influence of the length factor is small.

However, with the evolving process, the training accuracy difference becomes smaller than the threshold. The influence of hierarchical selection operator becomes bigger and shorter chromosome is selected first. In this way, the lengths of chromosomes becomes shorter gradually. Therefore, hierarchical selection operator controls bloat effect in a reasonable specific-to-general process.

Except the above two reasonable points, the hierarchical selection operator has a drawback that the training accuracy threshold is hard to set. If it is too big, the condition is easily satisfied and short chromosomes are selected with high probability. In this case, the system attach importance to the length factor excessively, which leads to the loss of diversity. The population can't evolve and premature convergence occures. On the other hand, if it is too small, then the condition is hard to satisfy and the chromosome with high training accuracy is selected more often. In this case, the length factor of the chromosome is ignored in fact and the sytem degenerates to GABIL system.

Taking the merits and demerits of the hierarchical selection operator into consideration, we improve on it first, and then reintroduce it to our system.

\subsection{Improvement on the operator}

Procedure:tune the threshold of the improved

hierarchical selection operator

Input:Maximum GA iterations( maxIterations ) ; fitness difference threshhold (threshold) ; continuous iterations (lastIterations) ; tune coefficient (a)

1. $\mathrm{i}=0$;

2. count $=0$

3. While $\mathrm{i}<$ maxIterations

4. Run GA

5. If the performance of the best

chromosome is improved

6 count $=0$;

7. Else

8. count ++ ;

9. End If

10. IF count $>$ lastIterations

11. threshold=threshold*a;

12. count $=0$;

13. i++;

14. End If

15. End While

Fig. 2: Tune the threshold of the improved hierarchical selection operator.

GAssist system uses MDL-based fitness function that considers the accuracy and complexity and also trades off the two factors. If hierarchical selection operator is introduced into GAssist system , the meaning of the threshold is the fitness difference rather than training accuracy difference. Besides, the threshold becomes easier to tune because of the change of fitness function. We only tune it in one direction. If the threshold is too small, then the fitness difference between two chromosomes isn't small than the threshold and hierarchical selection operator has no effect and chromosome with higher fitness value is selected. Assume threshold is 0, then the system goes back to GAssist system. In this case, although the improved hierarchical selection operator can't help to control 
bloat effect, it doesn't have any bad effect. Therefore, in this case the threshold could be very small and it becomes easier to tune.

In this paper,we tune the threshold by a heuristic process. First, set a threshold that is large enough to avoid premature convergence (experiments show that, 0.01 is safe [12]). With the iterating process going, if the performance of the best chromosome has not been improved after a continuous number of iterations, the threshold is tuned to smaller. This algorithm is descibed in Figure 2.

\section{The new stop criterion}

In general, we set the maximum number of iterations as the stop criterion, which is very simple, but inaccurate. If using generational replacement with elitism for the best chromosome, we can set the stop criterion according to the variation of the best chromosome [13]. GAssist system uses elitism selection, while using the maximum iterations as the stop criterion, so we propose that GA stops as the best chromosome has similar and stable performance through a certain number of iterations (MaximumBestDelayGA). The new stop criterion is as follows:

- The standard deviation of the accuracy for the last MaximumBestDelayGA iterations of the best chromosome is not larger than a threshold (thresh-accuracy).

- The difference of the maximum number of rules and the minimum number of rules of the best chromosome in the MaximumBestDelayGA iterations is not larger than a threshold (thresh-rule).

- The current iteration reaches the maximum number iterations.

If both the above two conditions are satisfied, GA stops iterating. If one of the condition is not satisfied GA doesn't stop running until it iterates the maximum number of iterations.

\section{Experiments}

\subsection{Experimental setup}

In this paper eight datasets are obtarined from UCI [14] machine learning repository. They are Iris, Glass, Hepatitis(Hepa), Hungarian(Hung), Balancescale(Bala), Credit-screening, Tic-Tac-Toe(Tic) and
Mushroom(Mush). The features of them are shown in Table 1.

Stratified ten-fold cross-validation [15] is used in experiments. In order to test all the four techniques proposed in this paper, experiments are conducted as the following analysis:

\begin{tabular}{|c|c|}
\hline Parameter & Value \\
\hline \multicolumn{2}{|l|}{ General parameters } \\
\hline Population size & 300 \\
\hline $\begin{array}{l}\text { Initial Number of rules in an } \\
\text { individual }\end{array}$ & 15 \\
\hline rule-wise Probability & 0.10 \\
\hline Selection Algorithm & Tournament \\
\hline Tournament size & 3 \\
\hline Fitness similarity thresh & 0.005 \\
\hline Crossover probability & 0.6 \\
\hline $\begin{array}{l}\text { Probability of mutating an } \\
\text { individual }\end{array}$ & 0.6 \\
\hline $\begin{array}{l}\text { Number of seeds for each } \\
\text { experiment }\end{array}$ & 15 \\
\hline $\begin{array}{l}\text { Probability of value } 1 \text { in } \\
\text { initialization }\end{array}$ & 0.75 \\
\hline $\begin{array}{l}\text { Number of incremental learning } \\
\text { segments }\end{array}$ & $2,3,15$ \\
\hline \multicolumn{2}{|l|}{ MDL Weight heuristically adjusting } \\
\hline Iteration of activation & 25 \\
\hline InitialRateOfComplexity & 0.075 \\
\hline MaximumBestDelay & 10 \\
\hline WeightRelaxationFactor & 0.9 \\
\hline \multicolumn{2}{|l|}{ Rule deletion operator } \\
\hline Iteration of activation & 5 \\
\hline $\begin{array}{l}\text { Minimum Number of rules before } \\
\text { disabling the operator }\end{array}$ & numClasses +3 \\
\hline \multicolumn{2}{|l|}{ fitness penalty } \\
\hline Iteration of activation & 25 \\
\hline Minimum No. of rules & Maximum-6 \\
\hline \multicolumn{2}{|c|}{ Improved Hierarchical Selection threshold adjusting } \\
\hline Initial value & 0.01 \\
\hline Iteration of activation & 40 \\
\hline MaximumBestDelaythresh & 10 \\
\hline ThreshRelaxationFactor & 0.9 \\
\hline \multicolumn{2}{|l|}{ The new stop criterion } \\
\hline Number of GA iterations & 1000 \\
\hline MaximumBestDelayGA & 10 \\
\hline thresh_accuracy & 0.005 \\
\hline thresh_rules & 2 \\
\hline
\end{tabular}

Table 3: Parameters of GA.

First, the performance of GAssist-Kohonen System is tested. Then the first technique is tested, if it is effective the second technique is introduced. In this way, four techniques should all be tested. Finally, we get five configurations that are detailed described in Table 2 .The detailed setting of parameters is shown in Table 3. 


\begin{tabular}{|l|l|l|l|}
\hline datasets & $\begin{array}{l}\text { Number of } \\
\text { examples }\end{array}$ & $\begin{array}{l}\text { Number of } \\
\text { attributes }\end{array}$ & $\begin{array}{l}\text { Number of } \\
\text { classes }\end{array}$ \\
\hline Iris & 150 & 5 & 3 \\
\hline Hepa & 155 & 20 & 2 \\
\hline Glass & 214 & 10 & 7 \\
\hline Hung & 294 & 14 & 2 \\
\hline Bala & 625 & 5 & 3 \\
\hline Cred & 690 & 16 & 2 \\
\hline Tic & 958 & 10 & 2 \\
\hline Mush & 8124 & 23 & 2 \\
\hline
\end{tabular}

Table1: feartures of datasets

\begin{tabular}{|l|l|}
\hline Code & Algorithm configuration \\
\hline 1 & GAssist-Kohonen \\
\hline 2 & 1 +new population initialization method \\
\hline 3 & 2+fitness scaling \\
\hline 4 & 3+improved hierarchical selection operator \\
\hline 5 & 4+new stop criterion \\
\hline
\end{tabular}

Table 2: Algorithm configurations.

\subsection{Experimental results and analysis}

Experimental results of five configurations are shown in Table 4. For each configuration and dataset we show the average value of: (1) the testing accuracy (Test Acc.), (2) the number of rules (No. of rules) (4) the running time in seconds. Analysing Table 4, we can get the following conclusions:

1) Testing accuracy is increased after the new population initialization technique is used while the size of the rule set and running time are nearly the same.

2) After calculating the fitness of chromosomes, fitness scaling is applied. Observing experimental results of configuration 2 and 3, we can see that the performance of the rule set is improved (the accuracy is increased or the size of rule set is reduced). Analysing in theory, fitness scaling can keep the competition of chromosomes in the population and promote the population's convergence. Therefore, the efficiency of the system should be improved, which has not been proved in experiments. It is because the new stop criterion has not been applied yet and GA has to run the maximum number of iterations.

3) Observing results of configuration 3 and 4, we can see that both the number of rules and the runtime are reduced significantly for all the datasets with none or only a little accuracy lose. Therefore, we can get a conclusion that our original objective of using the improved hierarchical selection operator to assist the MDL-based fitness function to control bloat effect has been achieved.

4) After the new stop criterion is used, three different results are observed:
- For Iris, Glass,Balance-scale and Mushroom, run-time is reduced significantly with none accuracy loses. In this case, the population has already converged to a global optimal solution, but the number of GA iterations hasn't been reached, so the new stop criterion works by stopping the searching process in time.

- For Hepatitis and Hungarian, although the running time is longer we get better solutions (increased average accuracy and reduced average number of rules). In this case, the population can still evolve, but the number of GA iterations is reached and the learning process is forced to stop. Because the new stop criterion contains stability of the performace of the best chromosome and large maximum iterations. Therefore, the new stop criterion going on to improve the performance by running more rounds.

- For the credit-screening and Tic-tac-toe, it suffers from over-fitting. In this case, the new stop criterion is too strict and GA can't search such a good solution, so it keeps running until reaching the maximum number of iterations which is setted a large number (1000).

Observing the above results, we can conclude that the four techniques are effective.

\section{Comparision with other symbolic learning methods}

There are many symbolic learning methods. For example, FCV, Classification based on Multiple Association Rules (CMAR), Bayes optimal classfier (Bayes) and C4.5. We compare our system with them. Experimental resulsts are shown in Table 5 and Table6.

Observing the results we can get the following conclusions:

- Because GA is a global search algorithm., comparing with other symbolic learning methods, it has a valuable merit that performs better for most datasets. Thus, we conclude that it can be used in more extensive questions.

- The efficiency of our system should be improved .

\section{Conclusions and further work}

In this paper, four techniques are proposed to improve GAssist system. First a new population initialization method is proposed and then fitness scaling is introduced into the 


\begin{tabular}{|l|l|l|l|l|l|}
\hline datasets & GA & FCV & CMAR & Bayes & C4.5 \\
\hline Iris & 98.8 & 83.1 & 93.3 & 74.2 & 94.4 \\
\hline Hepa & 85.5 & 87.6 & 81.5 & 87.1 & 76.0 \\
\hline Glass & 70.1 & 40.7 & 71.0 & 44.0 & 75.1 \\
\hline Hung & 84.2 & 91.0 & 83.3 & 83.9 & 77.1 \\
\hline Bala & 92.1 & 87.3 & 80.8 & 87.4 & 62.3 \\
\hline Cred & 84.3 & 91.6 & 84.9 & 86.3 & 85.5 \\
\hline Tic & 86.9 & 99.5 & 95.1 & 70.0 & 87.6 \\
\hline Mush & 98.4 & 100.0 & 96.2 & 99.5 & 100.0 \\
\hline
\end{tabular}

Table 5: Average accuracy of the global comparison experiments.

\begin{tabular}{|l|l|l|l|l|l|}
\hline datasets & GA & FCV & CMAR & Bayes & C4.5 \\
\hline Iris & 3.4 & 0.1 & 5.5 & 0 & 0 \\
\hline Hepa & 34.8 & 0.1 & 25.7 & 0 & 0 \\
\hline Glass & 7.7 & 0.1 & 60.1 & 0 & 0 \\
\hline Hung & 44.3 & 0.1 & 62.0 & 0 & 0 \\
\hline Bala & 21.71 & 0.2 & 0.1 & 0 & 0 \\
\hline Cred & 85.4 & 0.2 & 15.4 & 0 & 0 \\
\hline Tic & 73.4 & 0.2 & 4.5 & 0 & 0 \\
\hline Mush & 148.7 & 3.8 & 8.4 & 0 & 0 \\
\hline
\end{tabular}

Table 6: Average time of the global comparison experiments.

\begin{tabular}{|c|c|c|c|c|c|c|c|c|c|c|c|c|c|c|c|}
\hline \multirow[b]{2}{*}{ dataset } & \multicolumn{3}{|c|}{ Cofiguration 1} & \multicolumn{3}{|c|}{ Cofiguration 2} & \multicolumn{3}{|c|}{ Cofiguration 3} & \multicolumn{3}{|c|}{ Cofiguration 4} & \multicolumn{3}{|c|}{ Cofiguration 5} \\
\hline & $\begin{array}{l}\text { Test } \\
\text { Acc. }\end{array}$ & $\begin{array}{l}\text { No. } \\
\text { of } \\
\text { rule }\end{array}$ & $\begin{array}{l}\text { Time } \\
\text { (s) }\end{array}$ & $\begin{array}{l}\text { Test } \\
\text { Acc. }\end{array}$ & \#rule. & $\begin{array}{l}\text { Time } \\
\text { (s) }\end{array}$ & $\begin{array}{l}\text { Test } \\
\text { Acc. }\end{array}$ & $\begin{array}{l}\text { No. } \\
\text { of } \\
\text { rules }\end{array}$ & $\begin{array}{l}\text { Time } \\
\text { (s) }\end{array}$ & $\begin{array}{l}\text { Test } \\
\text { Acc. }\end{array}$ & $\begin{array}{l}\text { No. } \\
\text { of } \\
\text { rules }\end{array}$ & $\begin{array}{l}\text { Time } \\
\text { (s) }\end{array}$ & $\begin{array}{l}\text { Test } \\
\text { Acc. }\end{array}$ & $\begin{array}{l}\text { No. } \\
\text { of } \\
\text { rules }\end{array}$ & $\begin{array}{l}\text { Time } \\
\text { (s) }\end{array}$ \\
\hline Iris & 99.2 & 6.1 & 68.8 & 99.4 & 6.0 & 68.3 & 100.0 & 5.9 & 56.2 & 98.8 & 5.6 & 10.2 & 98.8 & 7.3 & 3.4 \\
\hline Нера & 85.1 & 6.2 & 38.2 & 85.6 & 6.2 & 37.3 & 85.9 & 6.2 & 39.8 & 83.9 & 5.7 & 13.2 & 85.5 & 6.0 & 34.8 \\
\hline Glass & 69.1 & 10.8 & 92.4 & 69.5 & 10.8 & 92.4 & 70.0 & 9.8 & 93.7 & 70.5 & 9.2 & 19.7 & 70.1 & 9.5 & 7.7 \\
\hline Hung & 82.9 & 11.6 & 12.5 & 83.2 & 11.4 & 12.4 & 83.7 & 6.9 & 51.6 & 82.6 & 6.2 & 10.1 & 84.2 & 6.1 & 44.3 \\
\hline Bala & 91.7 & 6.1 & 97.6 & 92.1 & 6.0 & 96.7 & 92.0 & 7.0 & 107.1 & 92.1 & 7.0 & 85.3 & 92.1 & 6.0 & 21.71 \\
\hline Cred & 87.5 & 7.8 & 30.6 & 88.2 & 7.8 & 29.6 & 87.0 & 6.3 & 38.5 & 87.8 & 6.0 & 13.2 & 84.3 & 5.2 & 85.4 \\
\hline Tic & 93.1 & 17.3 & 137.9 & 93.5 & 19.3 & 143.3 & 94.6 & 20.2 & 143.2 & 94.7 & 7.4 & 31.7 & 86.9 & 7.7 & 73.4 \\
\hline Mush & 99.5 & 5.0 & 268.6 & 99.7 & 5.0 & 263.6 & 99.4 & 5.4 & 267.0 & 99.4 & 3.5 & 234.9 & 98.4 & 5.7 & 148.7 \\
\hline
\end{tabular}
Table 4: Results of five algorithm configuration.

system. We also improve on the deserted hierarchical selection operator and combine it with the MDL-based fitness function to control bloat effect. Finally, a new stop criterion is applied. Experimental results show that for most datasets generalization capacity of the rule set and the efficiency are improved significantly. However, for a few datasets, the new stop criterion seems too strict, which leads to over-fitting. Therefore, the new stop criterion still needs improvement. Comparing with other symbolic learning methods, it is proved that GA has better performance for more extensive problems but the efficiency is still slow.

Therefore, as further work, we should study the way to fix the weakness of the new stop criterion. Besides, we should study new techniques to improve the efficiency of the system.

\section{Acknowledgement}

This work is financially supported by National Science Fund of China (60473045) and Hebei Province High Technology project (04213533).

\section{References}

[1] K.A. DeJong, W.M. Spears, Learning concept classifi-cation rules using genetic algorithms. Proceedings of the International Joint Conference on Artificial Intelligence, pp. 651656, 1991.
[2] K. A.DeJong, William M.Spears, Diana F.Gordon, Using genetic algorithms for concept learning. Machine Learning, 13(3):161-188, 1993.

[3] J. Bacardit, Pittsburgh genetics-based machine learning in the data mining era: representations, generalization, and run-time. Doctoral disertation, Ramon Llull University, Barcelona, Catalonia, Spain, 2004.

[4] J. Bacardit, N. Krasnogor, Smart crossover operator with multiple parents for a pittsburgh learning classifier system. Proceedings of the 8th Annual Conference on Genetic and Evolutionary Computation, pp. 1441-1448, 2006.

[5] J. Bacardit, N. Krasnogor, Empirical evaluation of ensemble techniques for a pittsburgh learning classifier system. Ninth International Workshop on Learning Classifier Systems,IWLCS2006, Lecture Notes in Artificial Intelligenge (to appear), Springer, 2006.

[6] T. Kohonen, Self-organization and associative memory. Springer, Berlin, 1988.

[7] C.T. Lin, C.S.G. Lee, Neural-network-based fuzzy logic control and decision system. IEEE Trans. Comput, 12: 1320-1336, 1991.

[8] J. Bacardit, Analysis of the initialization stage of a pittsburgh approach learning classifier system. Genetic and Evolutionary Computation Conference. Washington DC. pp. 1843-1850, 2005.

[9] J. Bacardit, David E. Goldberg, Martin V. Butz, Improving the performance of a pittsburgh 
learning classifier system using a default rule. Seventh International Workshop on Learning Classifier Systems, 2004.

[10]T. Kvan, Collaborative design: what is it? AUTOMATION IN CON2 STRACTION, 9(4): 409-415, 2000.

[11] W. B.Langdon, , Fitness causes bloat in variable sizerepresentations. Workshop on Evolutionary Co-mputation with Variable Size Representation at ICGA-97, May 141997.

[12] J. Bacardit., J. M. Garrell, Bloat control and generalization pressure using the minimum description length principle for a pittsburgh approach learning classifier system. Sixth International Workshop on Learning Classifier Systems Chicago, July 2003.

[13] M.Q. Li, D. Lin, Genetic algorithm's theory and application, Science Press, 2002.

[14] UCI repository of machine learning databases and domain theories, FTP address: ftp : // ftp. ics. uci. edu / pub / machine - learning - databases.

[15] R. Kohavi, A study of cross-validation and bootstrap for accuracy estimation and model selection. In International Joint Conference on Artificial Intelligence, pp.1137-1145, 1995. 